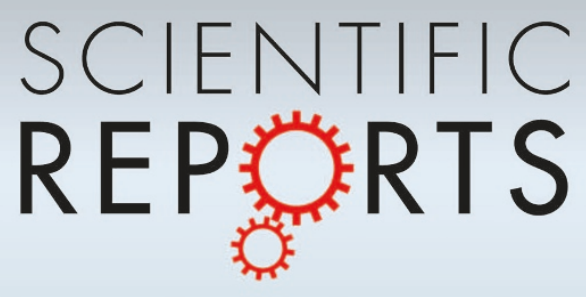

OPEN Quantum Corrections Crossover and

SUBJECT AREAS:

MATERIALS FOR DEVICES

ELECTRICAL AND ELECTRONIC

ENGINEERING

ELECTRONIC DEVICES

MAGNETIC PROPERTIES AND

MATERIALS

Received

24 April 2013

Accepted

15 July 2013

Published

9 August 2013

Correspondence and requests for materials should be addressed to

F.X. (Faxian@fudan. edu.cn)

* These authors contributed equally to this work.

\section{Ferromagnetism in Magnetic Topological Insulators}

\author{
Lihong Bao ${ }^{1,2 *}$, Weiyi Wang ${ }^{1 *}$, Nicholas Meyer ${ }^{2 *}$, Yanwen Liu' , Cheng Zhang' ${ }^{1}$ Kai Wang ${ }^{1,2}$, Ping Ai ${ }^{1}$ \\ \& Faxian Xiv'
} 'State Key Laboratory of Surface Physics and Department of Physics, Fudan University, Shanghai 200433, China, ${ }^{2}$ Department of
Electrical and Computer Engineering, lowa State University, Ames, IA 50010, USA.

Revelation of emerging exotic states of topological insulators (TIs) for future quantum computing applications relies on breaking time-reversal symmetry and opening a surface energy gap. Here, we report on the transport response of $\mathrm{Bi}_{2} \mathrm{Te}_{3} \mathrm{TI}$ thin films in the presence of varying $\mathrm{Cr}$ dopants. By tracking the magnetoconductance $(\mathrm{MC})$ in a low doping regime we observed a progressive crossover from weak antilocalization (WAL) to weak localization (WL) as the Cr concentration increases. In a high doping regime, however, increasing $\mathrm{Cr}$ concentration yields a monotonically enhanced anomalous Hall effect (AHE) accompanied by an increasing carrier density. Our results demonstrate a possibility of manipulating bulk ferromagnetism and quantum transport in magnetic TI, thus providing an alternative way for experimentally realizing exotic quantum states required by spintronic applications.

$\mathrm{n}$ recent years, topological insulators (TIs) have received considerable attention due to their novel properties arising from strong spin-orbit coupling and massless Dirac-cone-like surface states that are protected by timereversal symmetry (TRS) ${ }^{1-7}$. The exotic surface transport properties are manifested by the prohibition of backscattering events upon non-magnetic perturbations ${ }^{8,9}$ and the enhanced quantum corrections of magnetoconductance (MC), namely weak antilocalization (WAL) of Dirac fermions ${ }^{9-11}$. Breaking the TRS in TIs by interfacing a TI material with an insulating ferromagnetic film ${ }^{12-14}$ or simply by magnetically doping ${ }^{15-18}$ allows for the opening of a surface energy gap and the generation of massive surface carriers ${ }^{16}$. A variety of exotic properties can be realized including the topological magnetoelectric effect $^{18-20}$, the quantized anomalous Hall effect $^{18,21-23}$, imaging magnetic monopoles ${ }^{24}$, and the Faraday \& Kerr effects in TIs ${ }^{25,26}$. Revelation of these topologically non-trivial states calls for a comprehensive understanding of electronic transport response of magnetic doping in TIs.

One signature of such transport response is that when the TI is magnetically doped, a weak localization (WL) effect will naturally emerge as a result of the TRS breaking and the surface state gap opening ${ }^{27-29}$. By tuning the size of the opened surface gap or by moving the position of Fermi energy, the quantum corrections of MC experience a crossover from WAL to a conventional parabolic dependence of magnetic field and finally to WL, which were verified both theoretically ${ }^{27}$ and experimentally ${ }^{28}$. It has been shown that substituting the $\mathrm{Bi}$ sites in parent $\mathrm{Bi}$ based tetradymite compounds $\left(\mathrm{Bi}_{2} \mathrm{Se}_{3}, \mathrm{Bi}_{2} \mathrm{Te}_{3}, \mathrm{Sb}_{2} \mathrm{Te}_{3}, \mathrm{Bi}_{2-\mathrm{x}} \mathrm{Sb}_{\mathrm{x}} \mathrm{Te}_{3}\right.$, and $\left.\mathrm{Bi}_{2} \mathrm{Se}_{3-\mathrm{x}} \mathrm{Te}_{\mathrm{x}}\right)$ by transition metal ions $(\mathrm{Cr}$, $\mathrm{Fe}, \mathrm{Mn}, \mathrm{V}$, etc.) will produce a long-range ferromagnetic order either by coupling of local magnetic moments with bulk electron spins through Van Vleck mechanism ${ }^{21,30}$ or by Dirac fermion mediation of local magnetic moments via Ruderman-Kittel-Kasuya-Yosida (RKKY) exchange mechanism ${ }^{15,17,31,32}$. A hallmark of the presence of such ferromagnetism is anomalous Hall effect ${ }^{33}$, where Hall resistance shows a hysteresis loop behavior under magnetic field ${ }^{30,31,34}$. Observations of TRS breaking and surface gap opening by angle-resolved photoemission spectroscopy (ARPES) ${ }^{16,35-40}$ accelerate the investigations of transport properties in the magnetically doped TIs. Recently the Dirac fermion mediated ferromagnetism via RKKY interaction was observed in Mn-doped $\mathrm{Bi}_{2} \mathrm{Te}_{3-\mathrm{y}} \mathrm{Se}_{\mathrm{y}}$ nanpoflakes ${ }^{31}$ and carrier-independent long range ferromagnetic order was confirmed in Cr-doped $\mathrm{Bi}_{2-\mathrm{x}} \mathrm{Sb}_{\mathrm{x}} \mathrm{Te}_{3}$ thin films ${ }^{30}$, in which a large Van Vleck-type of spin susceptibility was established between local magnetic moments and bulk electrons that guarantees the robust ferromagnetism. However, to date, the tuning of ferromagnetism upon different dopant concentrations has not yet been demonstrated and the doping effect on the ferromagnetism needs to be further explored in the entire composition range. Recent experimental 
observation of quantum anomalous Hall effect in a magnetic TI Crdoped $\mathrm{Bi}_{2-\mathrm{x}} \mathrm{Sb}_{\mathrm{x}} \mathrm{Te}_{3}$ thin films also highlighted the role of different amount of dopants played in tuning the transport properties ${ }^{23}$.

In the present study, we report on the manipulation of ferromagnetism and quantum corrections in $\mathrm{Cr}_{\mathrm{x}} \mathrm{Bi}_{2-\mathrm{x}} \mathrm{Te}_{3}$ thin films grown on mica by varying the $\mathrm{Cr}$ doping concentration. In the low doping regime $(\mathrm{x} \leq 0.14)$, the quantum corrections of $\mathrm{MC}$ experience a crossover from WAL to WL. Once the ferromagnetism emerges, the quantum corrections of MC are dominated by the WL effect. While in the high doping regime $(x \geq 0.14)$, a monotonic enhancement in anomalous Hall effect was observed upon increasing $\mathrm{Cr}$ doping concentration. Our results demonstrated a promising way to manipulate the transport behavior of magnetic topological insulators.

\section{Results}

Structural characterizations of $\mathrm{MBE}$ grown $\mathrm{Cr}_{\mathrm{x}} \mathrm{Bi}_{2-\mathrm{x}} \mathrm{Te}_{3}$ thin films. Using molecular beam epitaxy (MBE) with high purity source materials, 15 quintuple layers (QLs) $\mathrm{Cr}_{\mathrm{x}} \mathrm{Bi}_{2-\mathrm{x}} \mathrm{Te}_{3}$ thin films were grown on muscovite mica via van der Waals epitaxy under a large range of dopant concentrations, where the Cr cell temperature was varied from 1020 to $1220^{\circ} \mathrm{C}$. During the growth the surface quality was in-situ monitored using reflection high-energy electron diffraction (RHEED) technique. Figs. 1a-c show the representative AFM images of as-grown pure (Fig. 1a) and Cr-doped (Figs. $1 \mathrm{~b}$ and 1c) $\mathrm{Bi}_{2} \mathrm{Te}_{3}$ thin films. The pure $\mathrm{Bi}_{2} \mathrm{Te}_{3}$ films have an atomically flat surface with micrometer-sized terraces, indicating the high crystalline quality (Fig. 1a). RHEED was used to monitor the insitu growth dynamics with the electron beam incident to the $[11 \overline{2} 0]$ direction. The sharp streaky lines in the inset of Fig. 1a indicate a layer-by-layer 2D growth mode and a flat surface morphology. Lightly doping with $\mathrm{Cr}(\mathrm{x}=0.08)$ resulted in the formation of triangular-shaped terraces without roughening the flat surface (Fig. 1b). While heavily doping with $\mathrm{Cr}(\mathrm{x}=0.27)$ roughened the surface with a root-mean square (RMS) roughness of $\sim 1 \mathrm{~nm}$. Previous studies on the Cr-doped TI materials $\mathrm{Sb}_{2} \mathrm{Te}_{3}$ and $\mathrm{Bi}_{2} \mathrm{Se}_{3}$ with rhombohedral symmetry have shown a tendency for $\mathrm{Cr}$ to be incorporated into the $\mathrm{Sb}$ or Bi sublattice ${ }^{41,42}$. The $\mathrm{Cr}$ in $\mathrm{Bi}_{2} \mathrm{Te}_{3}$ would likely be incorporated into $\mathrm{Bi}$ sites of the quintuple layer, which is schematically shown in Fig. 1d. The roughened surface of heavily Crdoped $\mathrm{Bi}_{2} \mathrm{Te}_{3}$ thin films is probably due to the competition between $\mathrm{Cr}$ atoms and $\mathrm{Bi}$ atoms at $\mathrm{Bi}$ sites in quintuple layered structure during the growth ${ }^{34}$. The high quality MBE grown thin films facilitate the revelation of transport response of $\mathrm{Cr}$ doping in $\mathrm{Bi}_{2} \mathrm{Te}_{3}$.

Crossover of quantum corrections in lightly doped $\mathrm{Cr}_{\mathbf{x}} \mathrm{Bi}_{2-\mathrm{x}} \mathrm{Te}_{3}$ thin films $(\mathbf{x} \leq \mathbf{0 . 1 4})$. The Cr-doped $\mathrm{Bi}_{2} \mathrm{Te}_{3}$ films on mica were etched into the Hall bar geometry using reactive ion etching (RIE) and the low temperature transport measurements in the longitudinal and transverse directions were carried out with a physical properties measurement system (PPMS) when a current is applied along the Hall bar and a magnetic field is applied perpendicularly to the surface. The longitudinal MC at low temperatures for a series of $\mathrm{Cr}$ doping concentrations is shown in Figure 2. We see evidences of the incorporation of $\mathrm{Cr}$ into the lattice and its effect on the transport for low $\mathrm{Cr}$ doping concentrations $(\mathrm{x} \leq 0.14)$ via quantum corrections. In pure $\mathrm{Bi}_{2} \mathrm{Te}_{3}$ thin films a sharp upward cusp is shown in the MC curve at low magnetic fields (Fig. 2a), indicating a WAL behavior ${ }^{9-11,43}$. This has been identified as a key feature of topological surface states, where Dirac fermions travel around a self-intersecting path or loop due to the spins rotating in opposite ways for the different path
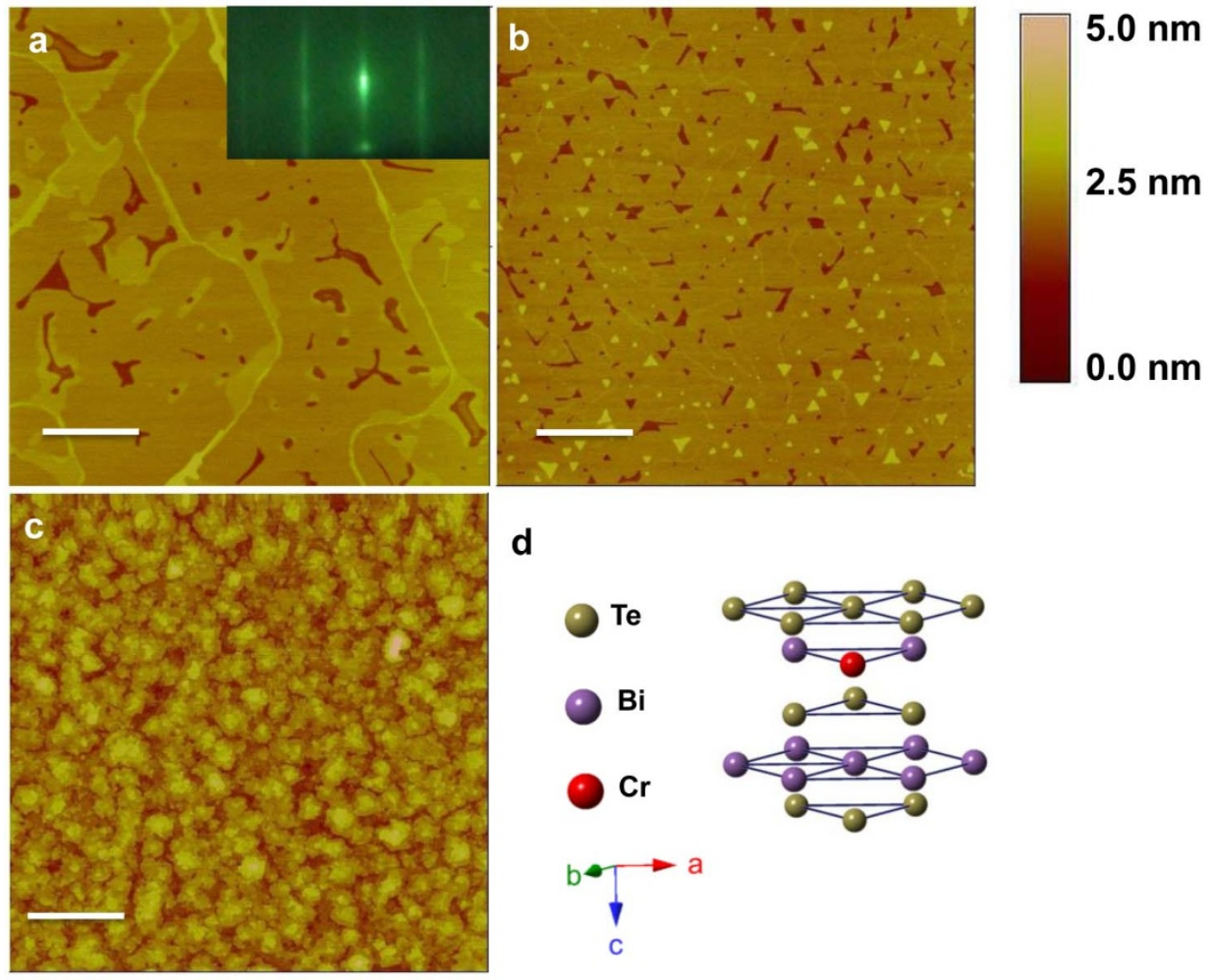

d
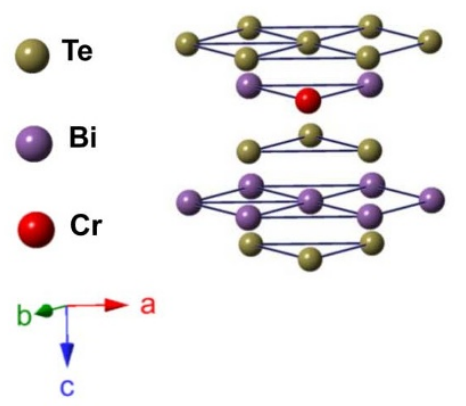

Figure 1 Structural characterizations of MBE grown $\mathrm{Cr}_{\mathrm{x}} \mathrm{Bi}_{2-\mathrm{x}} \mathrm{Te}_{3}$ thin films on mica. (a) An AFM image of a pure $\mathrm{Bi}_{2} \mathrm{Te}_{3}(\mathrm{x}=0)$ thin film, indicating an atomically smooth surface and micrometer-sized terraces. Inset is a representative RHEED pattern from the smooth surface of $\mathrm{Bi}_{2} \mathrm{Te}_{3}$ thin films.

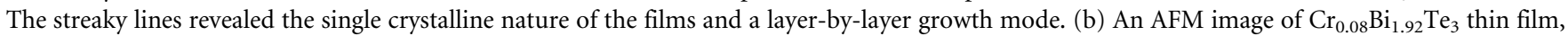
showing lightly doping of $\mathrm{Cr}$ induced the nucleation of triangular-shaped terraces on the smooth surface without roughening the surface. (c) An AFM image of $\mathrm{Cr}_{0.27} \mathrm{Bi}_{1.93} \mathrm{Te}_{3}$ thin film, demonstrating heavily doping of Cr roughed the smooth surface with an average surface roughness of $\sim 1 \mathrm{~nm}$.

(d) Atomically structural model of $\mathrm{Cr}_{\mathrm{x}} \mathrm{Bi}_{2-\mathrm{x}} \mathrm{Te}_{3}$, showing the position of $\mathrm{Cr}$ impurities is located at $\mathrm{Bi}$ sites of the quintuple layer. Scale bars are $1 \mu \mathrm{m}$. 

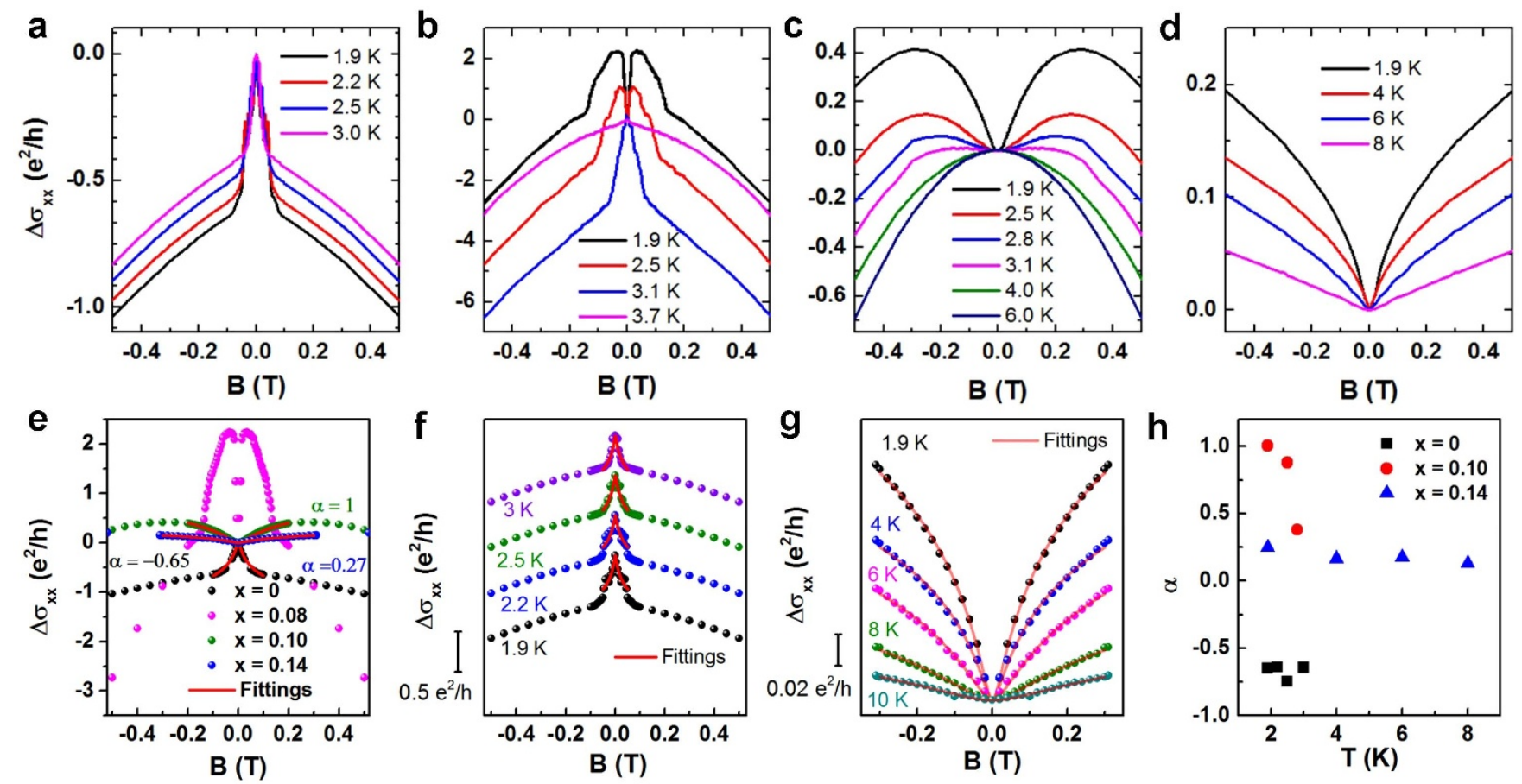

Figure $2 \mid$ Crossover of quantum corrections of magnetoconductance (MC) with increasing $\mathrm{Cr}$ content in $\mathrm{Cr}_{\mathrm{x}} \mathrm{Bi}_{2-\mathrm{x}} \mathrm{Te}_{3}$ thin films $(\mathrm{x} \leq 0.14)$. (a) $\mathrm{MC}$ curves of pure $\mathrm{Bi}_{2} \mathrm{Te}_{3}$ thin films, showing the negative $\mathrm{MC}$ features of WAL. (b) MC curves of $\mathrm{Cr}_{0.08} \mathrm{Bi}_{1.92} \mathrm{Te}_{3}$ thin film, indicating a nonmonotonic behavior with sharp downward cusp at low temperatures $(<3.1 \mathrm{~K})$ and re-presence of WAL at higher temperatures (3.1 and 3.7 $\mathrm{K}$ ). (c) $\mathrm{MC}$ curves of $\mathrm{Cr}_{0.10} \mathrm{Bi}_{1.90} \mathrm{Te}_{3}$ thin film, showing a crossover from downward cusp feature to parabolic dependence with increasing temperatures. (d) $\mathrm{MC}$ curves of $\mathrm{Cr}_{0.14} \mathrm{Bi}_{1.86} \mathrm{Te}_{3}$ thin film shows a WL dominated behavior. (e) HLN model fitting of MC curves with different $\mathrm{Cr}$ content at temperature of $1.9 \mathrm{~K}$. (f) and (g) HLN model fitting of MC curves of pure $\mathrm{Bi}_{2} \mathrm{Te}_{3}$ and heavily doped $\mathrm{Cr}_{0.14} \mathrm{Bi}_{1.86} \mathrm{Te}_{3}$ thin films, showing that both WAL and WL can be fitted well to the HLN model. (h) Pre-factor of $\alpha$ in HLN model of thin films with different Cr concentrations.

directions and a $\pi$ Berry's phase is accumulated ${ }^{10,11,44}$. The destructive interference due to $\pi$ Berry's phase leads to an enhancement of MC. Applying an external magnetic field suppresses the destructive interference, giving rise to a negative $\mathrm{MC}^{11,44,45}$. One interesting question to ask is what if the magnetic impurities are incorporated into the TI materials? Theoretical predictions suggested that when doping with magnetic impurities, a competing WL effect will be introduced and the localization behavior is a result of the competition between WAL and $\mathrm{WL}^{27,29,46,47}$. For the slightly doped sample $(\mathrm{x}=0.08$, Fig. $2 \mathrm{~b})$, at $\mathrm{T}=1.9 \mathrm{~K}$ the sharp upward cusp feature of WAL is gone and the magnetoconductance exhibits a non-monotonic increase with the increase of magnetic field, where a sharp downward cusp is developed at small magnetic fields. When the temperature warms up to $3.1 \mathrm{~K}$, the non-monotonic behavior disappears and the WAL shows up again. Further increasing the temperature to $3.7 \mathrm{~K}$ flattens the cusp feature, indicating that the WAL is weakened and it can only survive in a small temperature range. For the sample doped with $\mathrm{x}=0.10$ (Fig. $2 \mathrm{c}$ ), WAL is completely suppressed and a non-monotonic behavior is presented up to $3.1 \mathrm{~K}$ before a classical parabolic dependence of the magnetic field $\left(\sim \mathrm{B}^{2}\right)$ of the MC appears around $4 \mathrm{~K}$ (Supplementary materials Fig. S1). Further increasing the doping concentration to $\mathrm{x}=0.14$ (Fig. 2d), downward cusp feature is persistent up to $10 \mathrm{~K}$, indicative of a WL dominated behavior.

The quantum corrections to the $2 \mathrm{D}$ MC can be described by the Hikami-Larkin-Nagaoka (HLN) model $^{48}$ and is given analytically by the equation $\Delta \sigma_{x x} \equiv \sigma_{x x}(B)-\sigma_{x x}(0)=\alpha \frac{e^{2}}{\pi h}\left[\psi\left(\frac{1}{2}+\frac{B_{\phi}}{B}\right)-\right.$ $\left.\ln \left(\frac{B_{\phi}}{B}\right)\right]$, where $e$ is the electron charge, $h$ is Planck's constant, $B$ is the magnetic field, $\psi$ is the digamma function, and $\alpha$ is a coefficient whose value is determined by the nature of the corrections being WL or WAL, or having contributions from both effects. Additionally, we have $B_{\phi}=\hbar / 4 e l_{\phi}^{2}$ in which the coherence length is characterized by $l_{\phi}=\sqrt{D \tau_{\phi}}, D$ is the diffusion coefficient and $\tau_{\phi}$ is the dephasing time. The undoped samples show a WAL behavior (Fig. 2a) and can be fitted well to the HLN model (Figs. 2e and 2f). The resultant $\alpha$ value ranges from -0.65 to -0.75 (black squares in Fig. $2 \mathrm{~h}$ ) with increasing temperatures, consistent with the typical values of WAL originated from $2 \mathrm{D}$ surface states of $\mathrm{TI}^{11,49-51}$. And for the heavily doped samples with $\mathrm{x}=0.14$, the MC has an excellent fit to the HLN model (Fig. $2 \mathrm{~g}$ ) with $\alpha$ values from 0.25 to 0.09 (blue triangles in Fig. $2 \mathrm{~h}$ ) suggesting a typical WL behavior ${ }^{28,51-53}$. However, the fit becomes challenging for the lightly $(\mathrm{x}=0.08$, Fig. $2 \mathrm{~b})$ and intermediate doping $(x=0.10$, Fig. $2 \mathrm{c})$ samples, primarily because of the competition between WAL and WL. Under these circumstances, the weight ratios of competing terms of WAL and WL are difficult to be extracted. Nevertheless, in low magnetic fields $(-0.3 \mathrm{~T}<\mathrm{B}<$ $0.3 \mathrm{~T}$ ), the sample with intermediate doping yields $\alpha$ values ranging from 1.0 to 0.37 with increasing temperatures $(\mathrm{T} \leq 2.8 \mathrm{~K}$ ) as opposed to a large deviation from the HLN model at high fields (for B $>0.3 \mathrm{~T}$, supplementary Fig. S1) ${ }^{27}$.

It has been proposed that the opening of the surface energy gap from the TRS breaking is responsible for this crossover from WAL to $\mathrm{WL}^{27,28}$. Experimental observation in $\mathrm{Cr}_{\mathrm{x}} \mathrm{Bi}_{2-\mathrm{x}} \mathrm{Te}_{3}$ thin films showed that with $\mathrm{x}=0.23$, the surface states were completely suppressed. Correspondingly the system became a dilute magnetic semiconductor (DMS ${ }^{28}$. It is well known that the incorporation of magnetic impurities leads to the increased disorder in the films causing localization in the electronic states, known as WL, which is strongly related to field-induced magnetization ${ }^{29}$. In our scenario, with a much lower Cr doping of $\mathrm{x}=0.14$, the MC is completely governed by the WL effect as opposed to the crossover behavior from WL to unitary parabola with $\mathrm{x}=0.10$. This suggests that a long-range 
ferromagnetic order is developed upon the alignment of magnetic moments at low temperatures and low magnetic fields ${ }^{34}$.

Ferromagnetism in heavily doped $\mathrm{Cr}_{\mathrm{x}} \mathrm{Bi}_{2-\mathrm{x}} \mathrm{Te}_{3}$ thin films $(\mathrm{x} \geq$ 0.14). The Hall measurements were carried out to investigate the ferromagnetism in the $\mathrm{Cr}_{\mathrm{x}} \mathrm{Bi}_{2-\mathrm{x}} \mathrm{Te}_{3}$ films. In general, the Hall resistance of our samples doesn't show anomaly or hysteresis behavior until the doping level $\mathrm{x}$ reaches 0.14 . As shown in Figure 3, the Hall resistance $\mathrm{R}_{\mathrm{yx}}$ displays hysteresis loops resulting from anomalous Hall effect (AHE) at low temperatures ${ }^{33}$, showing a signature of a long-range ferromagnetism ${ }^{30,31,34}$. The Hall resistivity in a magnetic sample is given by $\rho_{y x}=R_{y x} d=R_{H} B+\rho_{A H}(M)$, where the first term is the ordinary Hall resistivity and the second term is the anomalous Hall contribution that arises from the magnetization of the material. Here, $d$ is the thickness of the film, $B$ is the applied magnetic field (in Tesla), $M$ is the magnetization, and $R_{H}$ is the ordinary Hall coefficient. Figs. 3 a-d show quasi-rectangular shaped hysteresis loops of $\mathrm{Cr}_{\mathrm{x}} \mathrm{Bi}_{2-\mathrm{x}} \mathrm{Te}_{3}$ thin films at low temperatures with $\mathrm{x}=0.14,0.27,0.30,0.32$, respectively. Both the saturation Hall resistance and the magnetization switching field decrease with increasing temperature, which is commonly observed in ferromagnetic materials. Fig. $3 e$ shows the temperature-dependent $R_{y x}$ of $\mathrm{Cr}_{\mathrm{x}} \mathrm{Bi}_{2-\mathrm{x}} \mathrm{Te}_{3}$ films at zero magnetic field. The Curie temperature can be defined as the temperature at which $\mathrm{R}_{\mathrm{yx}}$ reduces to zero when $\mathrm{B}=$ $0 \mathrm{~T}^{30,31}$. However, $\mathrm{R}_{\mathrm{yx}}$ does not completely vanish (several of tenth Ohms) at the measured temperature range (Figs. $3 \mathrm{a}-\mathrm{e})$. Therefore, the Curie temperature cannot be simply inferred from the $\mathrm{R}_{\mathrm{yx}}-\mathrm{T}$ relationship. The remaining hysteresis behavior can be ascribed to the defect or impurity states from mica substrates, which was previously observed in $\mathrm{Mn}_{\mathrm{x}} \mathrm{Bi}_{2-\mathrm{x}} \mathrm{Te}_{3}$ thin films grown on GaAs substrate $^{34}$. Alternative approach to identify the Curie temperature $T_{c}$ is to use Arrott plots, where $R_{y x}^{2}$ is plotted against $B / R_{y x}$ and the extrapolated intercept is proportional to the saturation magnetization (Supplementary material Figure S4) ${ }^{31,34}$. The Curie temperature $T_{c}$ can be extracted when the intercept on the $R_{y x}^{2}$ axis goes to zero ${ }^{31}$, as is shown in Fig. 3f. Fig. 3h summarizes the Curie temperature $T_{c}$ as a function of the $\mathrm{Cr}$ doping concentration. The monotonic increase in $T_{c}$ suggests an enhanced $\mathrm{AHE}$ with $\mathrm{Cr}$ concentration. By examining the Hall resistivity at large magnetic fields where magnetization is saturated and the response of the resistivity to the magnetic field is linear, we can extract the carrier concentration of the samples using Hall coefficient $\left|R_{H}\right|=1 / e n$, where $e$ is the electron charge and $n$ is the carrier concentration. The sign of the ordinary Hall coefficient exclusively shows an $n$-type conductivity for all $\mathrm{Cr}$ doping concentrations. Fig. $3 \mathrm{~g}$ shows a general trend of the increase of the sheet carrier concentration as a function of $\mathrm{Cr}$ concentration. The high bulk carrier concentration of $10^{14} \sim 10^{15} \mathrm{~cm}^{-2}$ with $\mathrm{x}=0.30$ $\sim 0.32$ of $\mathrm{Cr}$ indicates that $\mathrm{Cr}$ doping generated free carriers in $\mathrm{Bi}_{2} \mathrm{Te}_{3}$. As previously reported, Cr-doped p-type $\mathrm{Sb}_{2} \mathrm{Te}_{3}$ crystal exhibited a reduced hole density compared with a pure $\mathrm{Sb}_{2} \mathrm{Te}_{3}$ crystal, suggesting an $n$-type doping nature of $\mathrm{Cr}^{54}$. The high doping concentration of $\mathrm{Cr}$ will inevitably induced the chemical potential disorder ${ }^{17}$ in the $\mathrm{Cr}_{\mathrm{x}} \mathrm{Bi}_{2-\mathrm{x}} \mathrm{Te}_{3}$ thin films due to the competition between $\mathrm{Cr}$ atoms and $\mathrm{Bi}$ atoms in occupying the $\mathrm{Bi}$ sites ${ }^{34}$. The high carrier concentration in $\mathrm{Cr}_{\mathrm{x}} \mathrm{Bi}_{2-\mathrm{x}} \mathrm{Te}_{3}$ thin films most likely results from this chemical potential disorder ${ }^{17}$.

As discussed above, once the ferromagnetic order is established throughout the film, the MC is dominated by the WL $(x=0.14)$. With increasing Cr concentration (Figure 4, (a) $\mathrm{x}=0.27$, (b) $\mathrm{x}=$ 0.30 , and $(\mathrm{c}) \mathrm{x}=0.32$ ), the MC curves show a hysteresis behavior at low temperatures and progressively evolved into trivial flat shape with increasing temperatures. The butterfly-shaped hysteresis loop is an indication of the ferromagnetism in these thin films ${ }^{31,34,55}$. The MC curves show a negative quantum correction at low magnetic fields ${ }^{31,34}$ and the two MC minima presented in each butterfly pattern correspond to the coercive force. Consistent with the AHE results, coercive force varies a small amount with $\mathrm{Cr}$ concentrations increasing from $\mathrm{x}=0.27$ to 0.32 at $1.9 \mathrm{~K}$. Both longitudinal and transverse transport measurements verify the establishment of a long range ferromagnetic order in the heavily doped $\mathrm{Cr}_{\mathrm{x}} \mathrm{Bi}_{2-\mathrm{x}} \mathrm{Te}_{3}$ thin films.
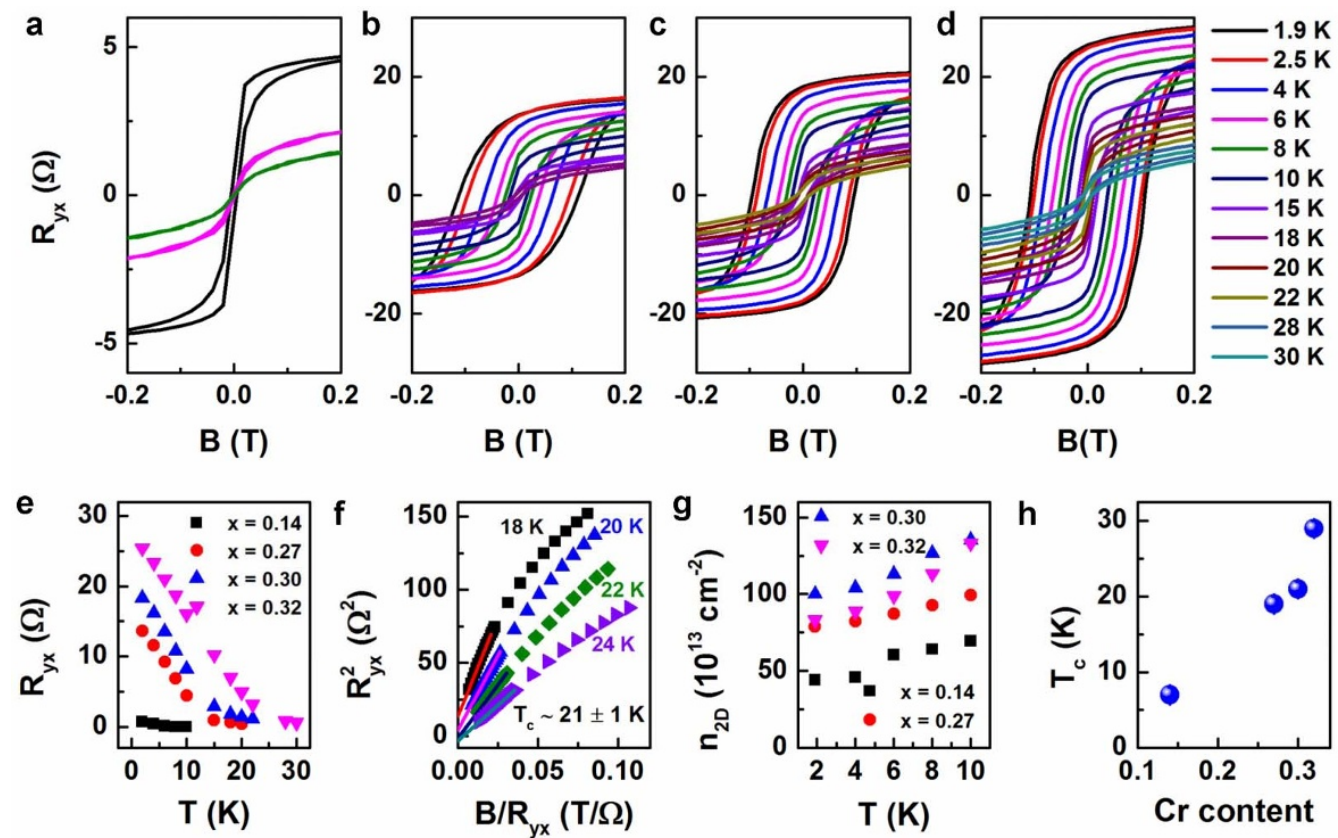

Figure 3 Anomalous Hall effect (AHE) in heavily doped $\mathrm{Cr}_{\mathrm{x}} \mathrm{Bi}_{2-\mathrm{x}} \mathrm{Te}_{3}$ thin films $(\mathrm{x} \geq \mathbf{0 . 1 4})$. Quasi-rectangular shaped hysteresis loop in magnetic field dependent Hall resistance curves at low magnetic fields $(\sim 0.2 \mathrm{~T})$ in $(\mathrm{a}) \mathrm{x}=0.14,(\mathrm{~b}) \mathrm{x}=0.27,(\mathrm{c}) \mathrm{x}=0.30$, and $(\mathrm{d}) \mathrm{x}=0.32$, showing that a ferromagnetic order is developed in these thin films. Increasing $\mathrm{Cr}$ concentration results in an enhancement of the AHE effect. (e) Temperature-dependent Hall resistances $\mathrm{R}_{\mathrm{yx}}$ of $\mathrm{Cr}_{\mathrm{x}} \mathrm{Bi}_{2-\mathrm{x}} \mathrm{Te}_{3}$ thin films at zero magnetic fields. (f) The Arrott plot of the $\mathrm{Cr}_{0.30} \mathrm{Bi}_{1.70} \mathrm{Te}_{3}$ thin film, showing the polarity change of intercept with increasing temperatures, by which the Curie temperature can be extracted. (g) Temperature-dependent carrier concentration of $\mathrm{Cr}_{\mathrm{x}} \mathrm{Bi}_{2-\mathrm{x}} \mathrm{Te}_{3}$ thin films. (h) Curie temperatures of $\mathrm{Cr}_{\mathrm{x}} \mathrm{Bi}_{2-\mathrm{x}} \mathrm{Te}_{3}$ thin films with different $\mathrm{Cr}$ concentration. 

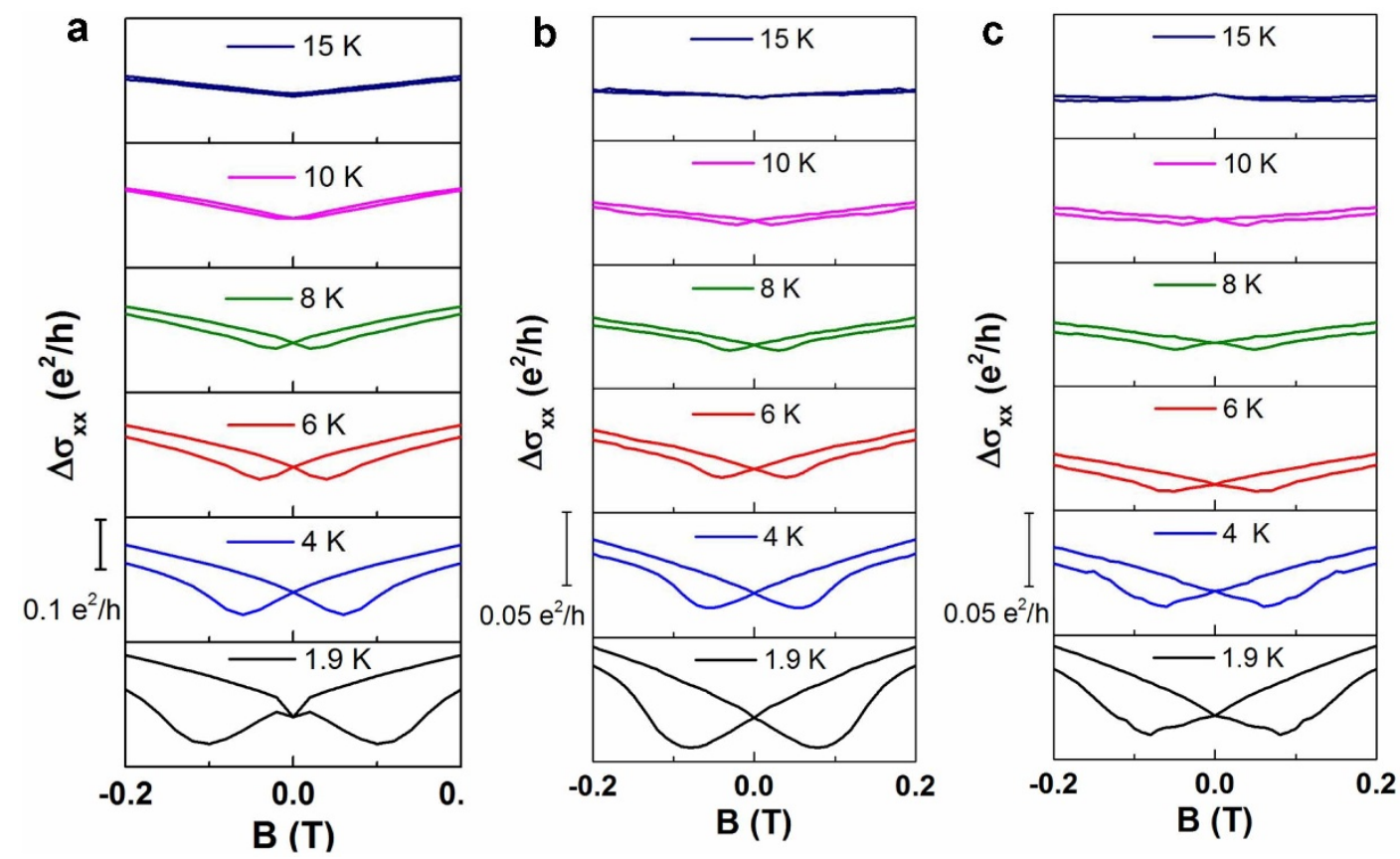

Figure $4 \mid$ Magnetoconductance (MC) curves of heavily doped $\mathrm{Cr}_{\mathrm{x}} \mathrm{Bi}_{2-\mathrm{x}} \mathrm{Te}_{3}$ thin films $(\mathrm{x} \geq \mathbf{0 . 1 4})$. The hysteresis loop behavior in $\mathrm{MC}$ curves in (a) $\mathrm{x}=$ 0.27 , (b) $\mathrm{x}=0.30$, and (c) $\mathrm{x}=0.32$, further confirming the establishment of ferromagnetism in these thin films. The MC minima in the MC curves reflect the strength of coercive force. Once $\mathrm{x}$ reaches 0.27 , the coercive force shows almost no response to the increasing $\mathrm{Cr}$ concentration.

However, the high carrier concentration observed in these thin films is an obstacle in obtaining the QAH state.

\section{Discussion}

In summary, mica serves as a suitable substrate to create high-quality flat surfaces for the TI material $\mathrm{Bi}_{2} \mathrm{Te}_{3}$. The incorporation of $\mathrm{Cr}$ dopants into the $\mathrm{Bi}_{2} \mathrm{Te}_{3}$ produces sufficient disorder to prompt a transition from WAL to WL in MC. The Hall resistivity shows hysteresis loops for Cr doping level $\mathrm{x} \geq 0.14$ due to the anomalous Hall effect, indicating that the film can become magnetized with a large $\mathrm{Cr}$ concentration. The results consolidate the idea that $\mathrm{Cr}$-doping is an appropriate approach to break TRS in the $\mathrm{Bi}_{2} \mathrm{Te}_{3}$ system as predicted in theoretical proposals ${ }^{21}$. The increased carrier concentration with increasing $\mathrm{Cr}$ concentration suggests that introducing $\mathrm{Cr}$ in $\mathrm{Bi}_{2} \mathrm{Te}_{3}$ thin films indeed generates free carriers. The high carrier concentration $\left(10^{14}-10^{15} \mathrm{~cm}^{-2}\right)$ in the ferromagnetic $\mathrm{Cr}_{\mathrm{x}} \mathrm{Bi}_{2-\mathrm{x}} \mathrm{Te}_{3}$ thin films eliminates the surface state transport and correspondingly rules out the possibility of Dirac fermion mediated RKKY mechanism. Previous experimental findings suggest that the Van Vleck mechanism is characterized by a carrier-independent long range ferromagnetic order ${ }^{30}$. Our experimental results didn't show a direct relation between the carrier density and ferromagnetism. Experimentally the other feature of Van Vleck mechanism is the linear relationship between Curie temperature and magnetic dopants concentration $^{30}$. However, our experiments didn't show a rigid linear relationship between Curie temperature and $\mathrm{Cr}$ doping concentration, as is shown in Fig. 3h. Our results demonstrate a clear crossover of quantum corrections of MC from WAL to WL at lightly doped regime $(\mathrm{x} \leq 0.14)$ and conventional butterfly patterned hysteresis loops at heavily doped regime $(x \geq 0.14)$. The transport signatures from the TRS-broken magnetic topological insulators in this study provides a critical reference for accessing of the gapped surface states, which is an important step toward the realization of novel topological magnetoelectric devices using non-trivial electronic states.

\section{Methods}

Thin film growth. High-quality crystalline thin films of $\mathrm{Cr}$-doped $\mathrm{Bi}_{2} \mathrm{Te}_{3}$ were grown on freshly cleaved muscovite mica via molecular beam epitaxy in an ultrahigh vacuum system with a base pressure of $\sim 10^{-10}$ Torr by co-evaporating high purity chromium (99.999\%), bismuth (99.999\%), and tellurium (99.999\%) sources under a Te rich condition. The films were obtained with a Bi cell temperature of $520^{\circ} \mathrm{C}$, a Te cell temperature of $320^{\circ} \mathrm{C}$, and a substrate temperature between 245 and $275^{\circ} \mathrm{C}$. Films were grown with various $\mathrm{Cr}$ concentrations by varying the $\mathrm{Cr}$ cell temperature from 1020 to $1230^{\circ} \mathrm{C}$. The thicknesses of thin films are determined by growth time and flux of $\mathrm{Bi}, \mathrm{Te}$, and $\mathrm{Cr}$ sources. Typical growth rate is $\sim 0.5 \mathrm{QL} / \mathrm{min}$.

Characterization. The morphology of as-grown $\mathrm{Cr}$-doped $\mathrm{Bi}_{2} \mathrm{Te}_{3}$ thin films was characterized with an atomic force microscope (AFM, Digital Instruments Nanoscope IIIa) and the Cr-doping profile was analyzed by an Oxford instrument Aztec X-ray energy-dispersive spectrum (EDS) system equipped on a field-emission gun SEM (FEI Quanta 250). At the Cr cell temperatures lower than $1160^{\circ} \mathrm{C}$, the yielding Cr doping profile is beyond the detection limit of EDS and the $\mathrm{Cr}$ concentrations were inferred from calibrated flux ratios of $\mathrm{Cr}$ cell and $\mathrm{Bi}$ cell combined with $\mathrm{EDS}^{56}$.

Device fabrication and transport measurement. Standard Hall bar devices of thin films were fabricated by photolithography combined with reactive ion etching (RIE). Ohmic contacts were established using room temperature cured silver paste. The transverse and longitudinal resistances were then measured using a Quantum Design physical properties measurement system (PPMS) that can sweep magnetic fields from $-9 \mathrm{~T}$ to $+9 \mathrm{~T}$ at temperatures as low as $1.9 \mathrm{~K}$.

1. Qi, X.-L. \& Zhang, S.-C. Topological insulators and superconductors. Rev. Mod. Phys. 83 (4), 1057-1110 (2011).

2. Hasan, M. Z. \& Kane, C. L. Colloquium: Topological insulators. Rev. Mod. Phys. 82 (4), 3045-3067 (2010).

3. Koenig, M. et al. Quantum spin hall insulator state in HgTe quantum wells. Science 318 (5851), 766-770 (2007).

4. Zhang, $\mathrm{H}$. et al. Topological insulators in $\mathrm{Bi}_{2} \mathrm{Se}_{3}, \mathrm{Bi}_{2} \mathrm{Te}_{3}$ and $\mathrm{Sb}_{2} \mathrm{Te}_{3}$ with a single Dirac cone on the surface. Nat. Phys. 5 (6), 438-442 (2009).

5. Chen, Y. L. et al. Experimental realization of a three-dimensional topological insulator, $\mathrm{Bi}_{2} \mathrm{Te}_{3}$. Science 325 (5937), 178-181 (2009).

6. Hsieh, D. et al. A topological Dirac insulator in a quantum spin Hall phase. Nature 452 (7190), 970-975 (2008).

7. Xiu, F. et al. Quantum capacitance in topological insulators. Sci. Rep. 2, 669 (2012).

8. Roushan, P. et al. Topological surface states protected from backscattering by chiral spin texture. Nature 460 (7259), 1106-1109 (2009).

9. He, H.-T. et al. Impurity effect on weak antilocalization in the topological insulator $\mathrm{Bi}_{2} \mathrm{Te}_{3}$. Phys. Rev. Lett. 106 (16), 166805 (2011).

10. Chen, J. et al. Gate-voltage control of chemical potential and weak antilocalization in $\mathrm{Bi}_{2} \mathrm{Se}_{3}$. Phys. Rev. Lett. 105 (17), 176602 (2010).

11. Bao, L. et al. Weak Anti-localization and Quantum Oscillations of Surface States in Topological Insulator $\mathrm{Bi}_{2} \mathrm{Se}_{2}$ Te. Sci. Rep. 2, 726; doi:10.1038/srep00726 (2012). 
12. Meng, Q., Vishveshwara, S. \& Hughes, T. L. Topological insulator magnetic tunnel junctions: Quantum Hall effect and fractional charge via folding. Phys. Rev. Lett. 109 (17), 176803 (2012).

13. Habe, T. \& Asano, Y. Interface metallic states between a topological insulator and a ferromagnetic insulator. Phys. Rev. B 85 (19), 195325 (2012).

14. Yokoyama, T., Tanaka, Y. \& Nagaosa, N. Anomalous magnetoresistance of a twodimensional ferromagnet/ferromagnet junction on the surface of a topological insulator. Phys. Rev. B 81 (12), 121401 (2010).

15. Liu, Q., Liu, C.-X., Xu, C., Qi, X.-L. \& Zhang, S.-C. Magnetic impurities on the surface of a topological insulator. Phys. Rev. Lett. 102 (15), 156603 (2009).

16. Chen, Y. L. et al. Massive Dirac fermion on the surface of a magnetically doped topological insulator. Science 329 (5992), 659-662 (2010).

17. Abanin, D. A. \& Pesin, D. A. Ordering of Magnetic Impurities and Tunable Electronic Properties of Topological Insulators. Phys. Rev. Lett. 106 (13), 136802 (2011).

18. Nomura, K. \& Nagaosa, N. Surface-quantized anomalous Hall current and the magnetoelectric effect in magnetically disordered topological insulators. Phys. Rev. Lett. 106 (16), 166802 (2011).

19. Qi, X.-L., Hughes, T. L. \& Zhang, S.-C. Topological field theory of time-reversal invariant insulators. Phys. Rev. B 78 (19) (2008).

20. Tse, W.-K. \& MacDonald, A. H. Magneto-optical and magnetoelectric effects of topological insulators in quantizing magnetic fields. Phys. Rev. B 82 (16), 161104 (2010).

21. Yu, R. et al. Quantized anomalous Hall effect in magnetic topological insulators. Science 329 (5987), 61-64 (2010).

22. Qi, X.-L., Hughes, T. L. \& Zhang, S.-C. Fractional charge and quantized current in the quantum spin Hall state. Nat. Phys. 4 (4), 273-276 (2008).

23. Chang, C.-Z. et al. Experimental observation of the quantum Anamolous Hall effect in a magnetic topological insulator. Science 340 (6129), 167-170 (2013).

24. Qi, X.-L., Li, R., Zang, J. \& Zhang, S.-C. Inducing a magnetic monopole with topological surface states. Science 323 (5918), 1184-1187 (2009).

25. Tse, W.-K. \& MacDonald, A. H. Magneto-optical Faraday and Kerr effects in topological insulator films and in other layered quantized Hall systems. Phys. Rev. B 84 (20), 205327 (2011)

26. Tse, W.-K. \& MacDonald, A. H. Giant magneto-optical Kerr effect and universal Faraday effect in thin-Film topological insulators. Phys. Rev. Lett. 105 (5), 057401 (2011).

27. Lu, H.-Z., Shi, J. \& Shen, S.-Q. Competition between weak localization and antilocalization in topological surface states. Phys. Rev. Lett. 107 (7), 076801 (2011).

28. Liu, M. et al. Crossover between weak antilocalization and weak localization in a magnetically doped topological insulator. Phys. Rev. Lett. 108 (3), 036805 (2012).

29. Cha, J. J. et al. Effects of magnetic doping on weak antilocalization in narrow $\mathrm{Bi}_{2} \mathrm{Se}_{3}$ nanoribbons. Nano Letters 12 (8), 4355-4359 (2012).

30. Chang, C.-Z. et al. Thin films of magnetically doped topological insulator with carrier-independent long-range ferromagnetic order. Adv. Mater. 25 (7), 1065-1070 (2013)

31. Checkelsky, J. G., Ye, J., Onose, Y., Iwasa, Y. \& Tokura, Y. Dirac-fermionmediated ferromagnetism in a topological insulator. Nat. Phys. 8 (10), 729-733 (2012).

32. Zhu, J.-J., Yao, D.-X., Zhang, S.-C. \& Chang, K. Electrically controllable surface magnetism on the surface of topological insulators. Phys. Rev. Lett. 106 (9), 097201 (2011)

33. Nagaosa, N., Sinova, J., Onoda, S., MacDonald, A. H. \& Ong, N. P. Anomalous Hall effect. Rev. Mod. Phys. 82 (2), 1539-1592 (2010).

34. Zhang, D. et al. Interplay between ferromagnetism, surface states, and quantum corrections in a magnetically doped topological insulator. Phys. Rev. B 86 (20), 205127 (2012)

35. Okada, Y. et al. Direct observation of broken time-reversal symmetry on the surface of a magnetically doped topological insulator. Phys. Rev. Lett. 106 (20), 206805 (2011).

36. Wray, L. A. et al. A topological insulator surface under strong Coulomb, magnetic and disorder perturbations. Nat. Phys. 7 (1), 32-37 (2011).

37. Caprara, S., Tugushev, V. V., Echenique, P. M. \& Chulkov, E. V. Spin-polarized states of matter on the surface of a three-dimensional topological insulator with implanted magnetic atoms. Phys. Rev. B 85 (12), 121304 (2012).

38. Scholz, M. R. et al. Tolerance of topological surface states towards magnetic moments: Fe on $\mathrm{Bi}_{2} \mathrm{Se}_{3}$. Phys. Rev. Lett. 108 (25), 256810 (2012).

39. Valla, T., Pan, Z. H., Gardner, D., Lee, Y. S. \& Chu, S. Photoemission spectroscopy of magnetic and nonmagnetic impurities on the surface of the $\mathrm{Bi}_{2} \mathrm{Se}_{3}$ topological insulator. Phys. Rev. Lett. 108 (11), 117601 (2012).
40. Xu, S.-Y. et al. Hedgehog spin texture and Berry's phase tuning in a magnetic topological insulator. Nat. Phys. 8 (8), 616-622 (2012).

41. Haazen, P. P. J. et al. Ferromagnetism in thin-film Cr-doped topological insulator $\mathrm{Bi}_{2} \mathrm{Se}_{3}$. Appl. Phys. Lett. 100 (8) (2012).

42. Kulbachinskii, V. A., Tarasov, P. M. \& Bruck, E. Ferromagnetism in a new dilute magnetic semiconductor $\mathrm{Sb}_{2-\mathrm{x}} \mathrm{Cr}_{\mathrm{x}} \mathrm{Te}_{3}$. J. Exp. Theor. Phys. 101 (3), 528-534 (2005).

43. Checkelsky, J. G., Hor, Y. S., Cava, R. J. \& Ong, N. P. Bulk band gap and surface state conduction observed in voltage-tuned crystals of the topological insulator $\mathrm{Bi}_{2} \mathrm{Se}_{3}$. Phys. Rev. Lett. 106 (19), 196801 (2011).

44. Matsuo, S. et al. Weak antilocalization and conductance fluctuation in a submicrometer-sized wire of epitaxial $\mathrm{Bi}_{2} \mathrm{Se}_{3}$. Phys. Rev. B 85 (7), 075440 (2012).

45. Cha, J. J. et al. Weak antilocalization in $\mathrm{Bi}_{2}\left(\mathrm{Se}_{\mathrm{x}} \mathrm{Te}_{1-\mathrm{x}}\right)_{3}$ nanoribbons and nanoplates. Nano Letters 12 (2), 1107-1111 (2012).

46. Lu, H.-Z. \& Shen, S.-Q. Weak localization of bulk channels in topological insulator thin films. Phys. Rev. B 84 (12), 125138 (2011).

47. Imura, K.-I., Kuramoto, Y. \& Nomura, K. Weak localization properties of the doped $\mathrm{Z}_{2}$ topological insulator. Phys. Rev. B 80 (8), 085119 (2009)

48. Hikami, S., Larkin, A. I. \& Nagaoka, Y. Spin-orbit interaction and magnetoresistance in the two dimensional random system. Prog. Theor. Phys. 63 (2), 707-710 (1980).

49. Kim, Y. S. et al. Thickness-dependent bulk properties and weak antilocalization effect in topological insulator $\mathrm{Bi}_{2} \mathrm{Se}_{3}$. Phys. Rev. B 84 (7), 073109 (2011).

50. Steinberg, H., Laloe, J. B., Fatemi, V., Moodera, J. S. \& Jarillo-Herrero, P. Electrically tunable surface-to-bulk coherent coupling in topological insulator thin films. Phys. Rev. B 84 (23), 233101 (2011).

51. Garate, I. \& Glazman, L. Weak localization and antilocalization in topologica insulator thin films with coherent bulk-surface coupling. Phys. Rev. B 86 (3), 035422 (2012).

52. Bergmann, G. Proximity effect in weak localization. Phys. Rev. Lett. 53 (11), 1100 (1984).

53. Altshuler, B. L., Aronov, A. G. \& Khmelnitsky, D. E. Effects of electron-electron collisons with small energy transfers on quantum localization. J. Phys. C 15 (36), 7367-7386 (1982).

54. Kulbachinskii, V. A., Tarasov, P. M. \& Bruck, E. Anomalous transport and ferromagnetism in the diluted magnetic semiconductor $\mathrm{Sb}_{2-\mathrm{x}} \mathrm{Cr}_{\mathrm{x}} \mathrm{Te}_{3}$. Physica $B$ 368 (1-4), 32-41 (2005).

55. Zhang, J. et al. Topology-driven magnetic quantum phase transition in topological insulators. Science 329, 1582-1586 (2013).

56. Zhang, J. et al. Band structure engineering in $\left(\mathrm{Bi}_{1-\mathrm{x}} \mathrm{Sb}_{\mathrm{x}}\right)_{2} \mathrm{Te}_{3}$ ternary topological insulators. Nat. Communi. 2 (2011)

\section{Acknowledgements}

This work is supported by the National Young 1000 Talents Plan, Shanghai Pu Jiang Talents plan, and US National Science Foundation under the Award No. 1201883.

\section{Author contributions}

F.X. conceived the idea and supervised the overall research. L.B. and N.M. designed and performed the experiments. N.M. and K.W. did the MBE growth of thin films. L.B. fabricated Hall bar devices. F.X., W.W., Y.L., C.Z. and P.A. carried out low-temperature transport measurements. L.B. and N.M. did the data analysis. L.B., N.M. and F.X. wrote the paper with helps from all other co-authors. L.B., W.W. and N.M. contributed equally to this work.

\section{Additional information}

Supplementary information accompanies this paper at http://www.nature.com/ scientificreports

Competing financial interests: The authors declare no competing financial interests.

How to cite this article: Bao, L.H. et al. Quantum Corrections Crossover and Ferromagnetism in Magnetic Topological Insulators. Sci. Rep. 3, 2391; DOI:10.1038/ srep02391 (2013)

This work is licensed under a Creative Commons Attribution 3.0 Unported license. To view a copy of this license, visit http://creativecommons.org/licenses/by/3.0 